\title{
Incorporating Erlotinib or Irinotecan Plus Cisplatin into Chemoradiotherapy for Stage III Non-small Cell Lung Cancer According to EGFR Mutation Status
}

\author{
Youngjoo Lee, MD, $\mathrm{PhD}{ }^{1}$ \\ Ji-Youn Han, MD, PhD' \\ Sung Ho Moon, MD2 \\ Byung-Ho Nam, $\mathrm{PhD}^{3}$ \\ Kun Young Lim, MD ${ }^{1}$ \\ Geon Kook Lee, MD, PhD' \\ Heung Tae Kim, MD, PhD ${ }^{1}$ \\ Tak Yun, MD \\ Hye Jin An, RN ${ }^{1}$ \\ Jin Soo Lee, MD, PhD ${ }^{1}$
}

${ }^{1}$ Center for Lung Cancer, National Cancer Center, Goyang, ${ }^{2}$ Center for Proton Therapy, National Cancer Center, Goyang,

${ }^{3}$ Center for Clinical Trials,

National Cancer Center, Goyang, Korea

Correspondence: Jin Soo Lee, MD, PhD

Center for Lung Cancer, National Cancer Center, 323 Ilsan-ro, Ilsandong-gu, Goyang 10408, Korea Tel: 82-31-920-1601

Fax: 82-31-920-1520

E-mail: jslee@ncc.re.kr

Received November 2, 2016

Accepted December 27, 2016

Published Online January 6, 2017

\begin{abstract}
Purpose
Concurrent chemoradiotherapy (CCRT) is the standard care for stage III non-small cell lung cancer (NSCLC) patients; however, a more effective regimen is needed to improve the outcome by better controlling occult metastases. We conducted two parallel randomized phase II studies to incorporate erlotinib or irinotecan-cisplatin (IP) into CCRT for stage III NSCLC depending on epidermal growth factor receptor (EGFR) mutation status.
\end{abstract}

\section{Materials and Methods}

Patients with EGFR-mutant tumors were randomized to receive three cycles of erlotinib first and then either CCRT with erlotinib followed by erlotinib (arm A) or CCRT with IP only (arm B). Patients with EGFR unknown or wild-type tumors were randomized to receive either three cycles of IP before (arm C) or after CCRT with IP (arm D).

\section{Results}

Seventy-three patients were screened and the study was closed early because of slow accrual after 59 patients were randomized. Overall, there were seven patients in arm A, five in arm B, 22 in arm C, and 25 in arm D. The response rate was $71.4 \%$ and $80.0 \%$ for arm $A$ and $B$, and $70.0 \%$ and $73.9 \%$ for arm $C$ and $D$. The median overall survival (OS) was 39.3 months versus 31.2 months for arm $A$ and $B(p=0.442)$, and 16.3 months versus 25.3 months for arm $C$ and $D(p=0.050$ ). Patients with sensitive $E G F R$ mutations had significantly longer OS than EGFR-wild patients ( 74.8 months vs. 25.3 months, $p=0.034)$. There were no unexpected toxicities.

\section{Conclusion}

Combined-modality treatment by molecular diagnostics is feasible in stage III NSCLC. EGFRmutant patients appear to be a distinct subset with longer survival.

\section{Key words}

Chemoradiotherapy, EGFR tyrosine kinase inhibitor, EGFR mutation, Non-small-cell lung carcinoma

\section{Introduction}

Lung cancer remains the leading cause of cancer death in both men and women worldwide [1], including in Korea [2]. Non-small-cell lung cancer (NSCLC) accounts for approximately $85 \%$ of all lung cancers, and $30 \%$ to $40 \%$ of these patients present with unresectable stage IIIA and IIIB disease [3]. For stage III NSCLC patients with a good performance status (PS), combined-modality therapy using chemotherapy and thoracic radiation therapy represents the standard of care. In addition, several phase III trials have demonstrated that the concurrent administration of these two modalities improves long-term survival relative to sequential strategies, resulting in the general acceptance of concurrent chemoradiotherapy (CCRT) as a vital component of standard therapeutic paradigms $[4,5]$. Despite this improved survival, more than two thirds of patients develop overt metastatic disease within 2 to 3 years, and most patients die from their disease as a result of distant failure. Therefore, control of occult 
micrometastatic disease remains a challenge in this patient population.

However, molecularly-targeted therapy using epidermal growth factor receptor tyrosine kinase inhibitors (EGFR-TKI) has brought new enthusiasm to the treatment of NSCLC patients over the last decade. While the initial clinical trials did not demonstrate a survival benefit with EGFR-TKIs for the overall population of advanced NSCLC patients [6], a specific subset of patients carrying mutations on the kinase domain of EGFR gene were found to be highly sensitive to the these drugs $[7,8]$. Encouraged by these results, we thought that if cases were properly selected, unresectable stage III NSCLC patients would benefit from EGFR-TKI treatment as good as those with stage IV NSCLC. Thus, we designed a randomized phase II trial to evaluate the efficacy and toxicity of EGFR-TKI erlotinib incorporated into the CCRT setting for patients with EGFR-mutant stage III NSCLC.

This study was also designed to develop an optimal treatment strategy to resolve occult micrometastatic disease by administering more effective chemotherapy at full dose before or after CCRT for patients with EGFR unknown or wild-type tumors. Earlier randomized trials that reported inferior outcomes for induction chemotherapy followed by radiotherapy (RT) utilized old chemotherapeutic agents such as vinblastine, vindesine, and mitomycin $[4,5]$. Therefore, we explored the impact of more effective irinotecan plus cisplatin (IP) regimen as induction or consolidation chemotherapy. We used the IP regimen because the clinical synergism of this combination was demonstrated in several clinical trials that reported $25 \%-59 \%$ response rates and 1-year survival rates as high as $57 \%$ for patients with advanced NSCLC [9-12]. We previously conducted a phase II study using IP regimen in patients with advanced NSCLC. Of 77 assessable patients, 36 achieved partial response (PR); thus, the overall response rate (ORR) was 47\% [13]. In our study, the median overall survival (OS) was 15.2 months (range, 2.9 to 29.7 months) with a 1-year survival rate of $63.3 \%$ (95\% confidence interval $[\mathrm{CI}], 52.4 \%$ to $74.1 \%$ ). Furthermore, IP regimen showed a more favorable toxicity profile than etoposide plus cisplatin (EP) in a phase III trial of chemo-naïve extensive stage small cell lung cancer [14]. Based on the favorable activity and toxicity profile, we selected IP regimen rather than EP for the concurrent treatment.

Collectively, we conducted two parallel randomized phase II studies to incorporate erlotinib or IP into CCRT for stage III NSCLC stratified by the EGFR mutation status.

\section{Materials and Methods}

\section{Eligibility}

Patients ( $\geq 18$ years of age) with a cytological or histological confirmation of NSCLC, unresectable stage IIIA (T1-3N2) confirmed by surgeon or stage-IIIB (T4N2 or T1-4N3 not malignant pleural effusion) according to the American Joint Committee on Cancer sixth edition, a PS of 0 or 1 on the Eastern Cooperative Oncology Group (ECOG) scale, and no prior therapy for NSCLC were eligible. Adequate tissue samples for EGFR mutation analysis were requested, but not mandatory. The qualifying laboratory criteria were as follows: absolute neutrophil counts of $\geq 2,000 / \mu \mathrm{L}$, platelets of $\geq 100,000 / \mu \mathrm{L}$, hemoglobin of $\geq 10 \mathrm{~g} / \mathrm{dL}$, total serum bilirubin of $\leq 1.0 \times$ the institutional upper limit of normal (ULN), serum transaminases of $\leq 2.0 \times \mathrm{ULN}$, and serum creatinine levels $\leq 1.0 \times$ ULN. Patients whose forced expiratory volumes in 1 second were lower than $1 \mathrm{~L}$ by spirometry were excluded. All the patients provided written informed consent, and this study was approved by the institutional review board (ClinicalTrials.gov, NCT00620269).

\section{Study design and treatment}

This was a single center, open-label, parallel, randomized, phase II study. The stratification factors were supraclavicular lymph node, histology (adenocarcinoma vs. non-adenocarcinoma), and smoking history.

Patients with $E G F R$-mutant tumors initially received three cycles of erlotinib and were then treated by CCRT with either two cycles of erlotinib (arm A) or IP (arm B) (Fig. 1). After completion of CCRT, the patients in arm A received consolidation therapy with six cycles of erlotinib, while those in arm $B$ were observed until disease progression and restarted erlotinib after progression. Patients with EGFR unknown or wild-type tumors were treated by either induction therapy with three cycles of IP followed by CCRT with two cycles of IP (arm C) or CCRT with two cycles of IP followed by consolidation therapy with three cycles of IP (arm D). Erlotinib was administered at $150 \mathrm{mg}$ daily with a 3-week cycle. IP was administered with cisplatin $30 \mathrm{mg} / \mathrm{m}^{2}$ (days 1 and 8) and irinotecan $60 \mathrm{mg} / \mathrm{m}^{2}$ (days 1 and 8) during RT or with cisplatin $30 \mathrm{mg} / \mathrm{m}^{2}$ (days 1 and 8 ) and irinotecan $65 \mathrm{mg} / \mathrm{m}^{2}$ (days 1 and 8) during induction or consolidation therapy with a 3-week cycle.

All thoracic RT was based on computed tomography (CT) planning. The CT simulation was performed with a GE LightSpeed RT (GE Healthcare, Milwaukee, WI) or Picker CT-Simulator UltraZ (Philips Medical System, Best, The Netherlands), and each scan slice had a thickness of $35 \mathrm{~mm}$. 


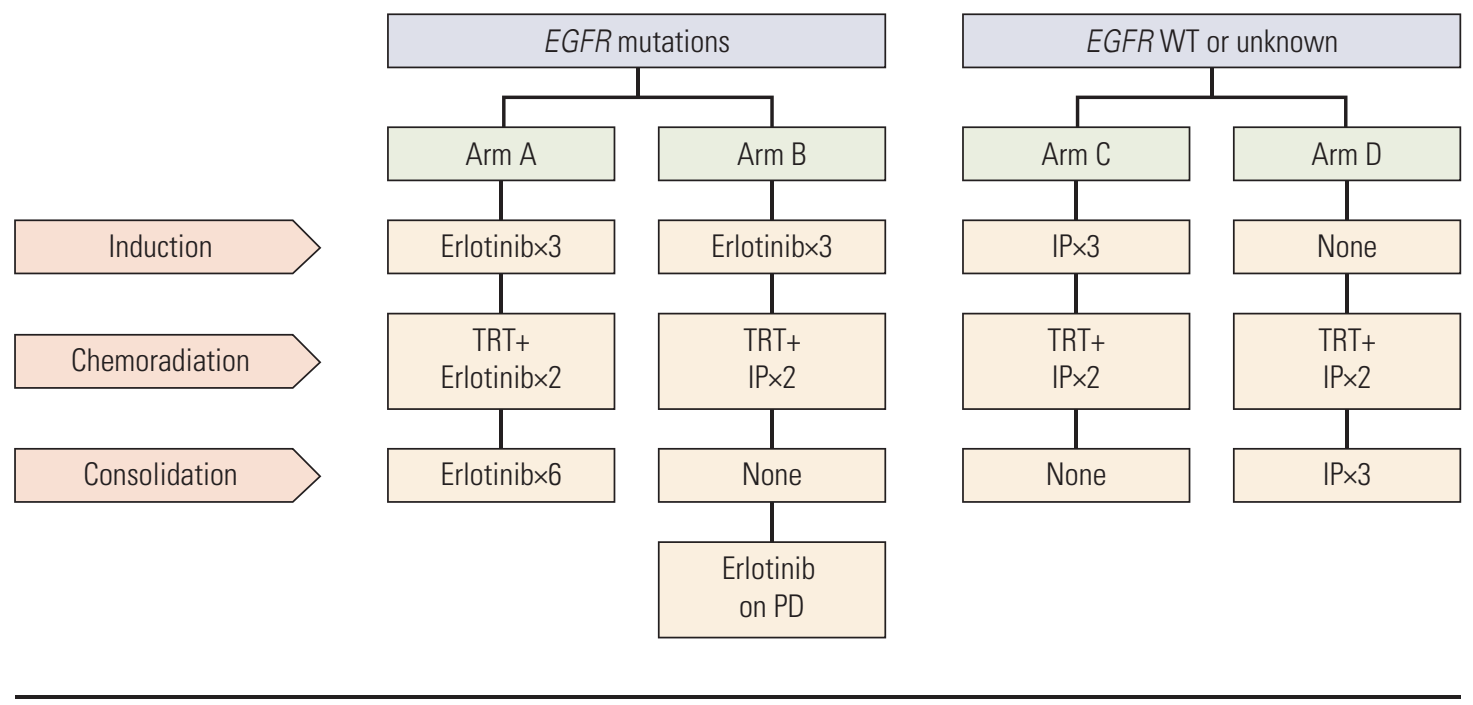

Erlotinib $150 \mathrm{mg}$ daily for 21 days

IP as induction/consolidation: irinotecan $65 \mathrm{mg} / \mathrm{m}^{2}+$ cisplatin $30 \mathrm{mg} / \mathrm{m}^{2}$ intravenously D1 \& D8 every 3 weeks

IP with TRT: irinotecan $60 \mathrm{mg} / \mathrm{m}^{2}+$ cisplatin $30 \mathrm{mg} / \mathrm{m}^{2}$ intravenously D1 \& D8 every 3 weeks

TRT: 60 Gy/30 fractions over 6 weeks

Fig. 1. Study design. EGFR, epidermal growth factor receptor; WT, wild type; IP, irinotecan plus cisplatin; TRT, thoracic radiotherapy; $\mathrm{PD}$, disease progression.

The target volumes were defined as follows: gross tumor volume (GTV), primary tumor(s) and involved lymph node(s); clinical target volume (CTV), GTV $+1 \mathrm{~cm}$ for microscopic tumor extension; planning target volume (PTV), CTV $+5 \mathrm{~mm}$ margin. A minimum of 36 coplanar isocentric fields were designed for 3-dimensional conformal radiotherapy (3D-CRT) and intensity modulated radiotherapy (IMRT) with pinnacle radiotherapy treatment planning (RTP) systems (Philips Radiation Oncology Systems, Milpitas, CA) or the Eclipse RTP system, ver. 8.3 (Varian Medical System Inc., Palo Alto, CA). A once daily dose of $2.4 \mathrm{~Gy}$ was delivered up to $60 \mathrm{~Gy}$ in 25 fractions for 5 days per week. The daily dose of $2.4 \mathrm{~Gy}$ was composed of 1.8 Gy to PTV and a concomitant boost dose of 0.6 Gy to GTV, with a field border defined as GTV+ $1-1.5 \mathrm{~cm}$ for 3D-CRT and planning GTV as GTV+8-10 mm considering set-up error and internal motion for IMRT.

\section{Patient evaluation}

A disease assessment by chest CT was conducted after three cycles of induction therapy, 4 weeks after completion of CCRT, and then every 8 weeks thereafter until disease progression. The ORR was determined by the number of patients with complete response and PR according to the Response Evaluation Criteria in Solid Tumor committee 1.0
[15]. Adverse events were evaluated according to the Common Terminology Criteria for Adverse Events ver. 3.0.

\section{Statistical analysis}

The primary endpoints are ORR, toxicities, and OS. The secondary endpoints are progression-free survival (PFS) and patterns of failure. The sample size calculation was as follows.

\section{1) For patients with EGFR-mutant tumor}

Assuming accrual of 50 patients over 2 years and 2 years of follow-up, this number of patients will provide a $73 \%$ power to detect a 7-month difference in median survival with a type I error of 0.05 based on a one-sided test (i.e., 17 months for the historical CCRT vs. 24 months for the EGFR mutation positive group as a whole). However, allowing for an invaluable rate of up to $10 \%$, the total sample size will be 56 patients (per each arm of 28 patients).

\section{2) For patients with EGFR unknown or wild-type tumor}

Assuming accrual of 148 patients over 2 years and 2 years of follow-up from the last accrual, with $80 \%$ power to detect 
Table 1. Patient characteristics

\begin{tabular}{|c|c|c|c|c|}
\hline \multirow[t]{2}{*}{ Characteristic } & \multicolumn{2}{|c|}{$\begin{array}{l}\text { EGFR mutant type } \\
\qquad(\mathrm{n}=12)\end{array}$} & \multicolumn{2}{|c|}{$\begin{array}{l}\text { EGFR wild type }(\mathrm{n}=28) \\
\text { or unknown }(\mathrm{n}=19)\end{array}$} \\
\hline & $\operatorname{Arm~A}(n=7)$ & Arm B $(n=5)$ & $\operatorname{Arm~C~(n=22)~}$ & $\operatorname{Arm} D(n=25)$ \\
\hline Age, median (range, yr) & $61(37-78)$ & $62(51-78)$ & $60(37-74)$ & $61(42-76)$ \\
\hline \multicolumn{5}{|l|}{ Sex } \\
\hline Female & $5(71.4)$ & $3(60.0)$ & $3(13.6)$ & $4(16.0)$ \\
\hline Male & $2(28.6)$ & $2(40.0)$ & $19(86.4)$ & $21(84.0)$ \\
\hline \multicolumn{5}{|l|}{ Smoking } \\
\hline Never & $5(71.4)$ & $3(60.0)$ & $2(9.1)$ & $3(12.0)$ \\
\hline Ever & $2(28.6)$ & $2(40.0)$ & $20(90.9)$ & $22(88.0)$ \\
\hline \multicolumn{5}{|l|}{ ECOG PS } \\
\hline 0 & $3(42.9)$ & $1(20.0)$ & $10(45.5)$ & $7(28.0)$ \\
\hline 1 & $4(57.1)$ & $4(80.0)$ & $12(54.5)$ & $18(72.0)$ \\
\hline \multicolumn{5}{|l|}{ Pathology } \\
\hline $\mathrm{ADC}$ & $6(85.7)$ & $5(100)$ & $8(36.4)$ & $9(36.0)$ \\
\hline Non-ADC & $1(14.3)$ & 0 & $14(63.6)$ & $16(64.0)$ \\
\hline \multicolumn{5}{|l|}{ Stage $^{\text {a) }}$} \\
\hline IIIA & $2(28.6)$ & $1(20.0)$ & $5(22.7)$ & $7(28.0)$ \\
\hline IIIB & $5(71.4)$ & $4(80.0)$ & $17(77.3)$ & $18(72.0)$ \\
\hline \multicolumn{5}{|l|}{ Supraclavicular LN } \\
\hline Involved & $2(28.6)$ & $2(40.0)$ & $12(54.5)$ & $11(44.0)$ \\
\hline Not involved & $5(71.4)$ & $3(60.0)$ & $10(45.5)$ & $14(56.0)$ \\
\hline
\end{tabular}

Values are presented as number (\%). EGFR, epidermal growth factor receptor; ECOG, Eastern Cooperative Oncology Group; PS, performance status; ADC, adenocarcinoma; LN, lymph node. ${ }^{a}$ Staging according to the seventh edition of American Joint Committee on Cancer TNM staging system.

a 7-months difference in median survival with a type I error of 0.05 based on a one-sided test (i.e., 17 months for the historical CCRT vs. 24 months for the EGFR mutation negative group with new treatment as a whole), we need 74 patients per arm. However, allowing for a follow-up loss rate of up to $5 \%$, the total sample size will be total 156 patients per each arm of 78 patients.

OS was calculated from the start date of study treatment to the date of death. PFS was calculated from the start date of study treatment to the first objective documentation date of progressive disease or death. Pearson's chi-square test or Fisher exact test was used to compare the ORR of treatment arms, where appropriate. Survival time was estimated using the Kaplan-Meier method, and survival difference between groups was assessed using the log-rank test.

\section{EGFR mutation analysis}

Nucleotide sequencing of the kinase domain of the EGFR gene (exons 19, 20, and 21) was conducted using nested polymerase chain reaction amplification of the individual exons. The details of the sequencing procedure have been described elsewhere [16]. When this study was initially designed, there was no information available regarding drug sensitivity for uncommon EGFR mutations; thus, patients having tumors with all types of EGFR mutation were allocated into arm A and $\mathrm{B}$.

\section{Results}

Recruitment started in March of 2008 and stopped in August of 2014 due to slow accrual rate. Overall, 73 patients were screened and 59 were enrolled. We analyzed EGFR mutations in tumor biopsy samples of 40 patients. There were 12 patients with EGFR-mutant tumors (exon 19 deletion in 10, exon 20 insertion in two) who were randomly assigned to arm $A(n=7)$ and arm $B(n=5)$, while 47 patients with 28 EGFR-wild and 19 EGFR-unknown tumors were assigned to $\operatorname{arm} C(\mathrm{n}=22)$ and arm D (n=25) (S1 Fig.). 
Table 2. ORR after concurrent chemoradiotherapy

\begin{tabular}{|c|c|c|c|c|c|}
\hline Response & $\begin{array}{c}\text { Arm A } \\
(n=7)\end{array}$ & $\begin{array}{c}\text { Arm B } \\
(n=5)\end{array}$ & $\begin{array}{l}\text { Arm C } \\
(n=22)\end{array}$ & $\begin{array}{r}\text { Arm D } \\
(n=25)\end{array}$ & p-value \\
\hline $\mathrm{CR}$ & 0 & 0 & 0 & 1 & \\
\hline PR & 5 & 4 & 14 & 16 & \\
\hline SD & 0 & 1 & 5 & 6 & \\
\hline PD & 2 & 0 & 1 & 0 & \\
\hline NE & 0 & 0 & 2 & 2 & \\
\hline ORR (CR+PR), n (\%) & $5 / 7(71.4)$ & $4 / 5(80.0)$ & $14 / 20(70.0)$ & $17 / 23(73.9)$ & 0.906 \\
\hline
\end{tabular}

$\mathrm{CR}$, complete response; $\mathrm{PR}$, partial response; $\mathrm{SD}$, stable disease; PD, disease progression; NE, not evaluable; ORR, overall response rate.

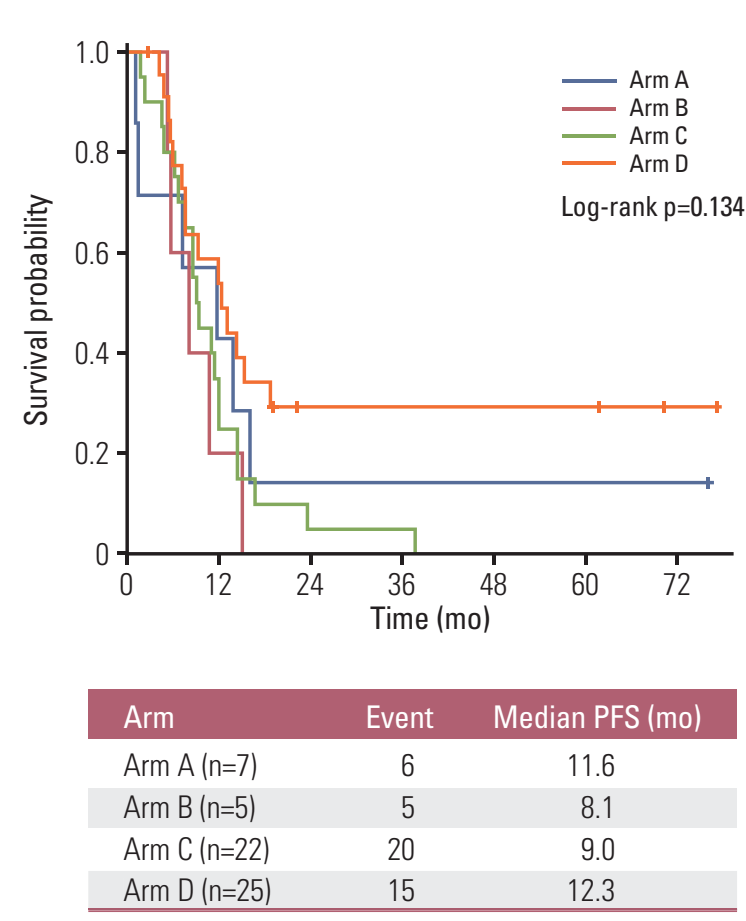

A

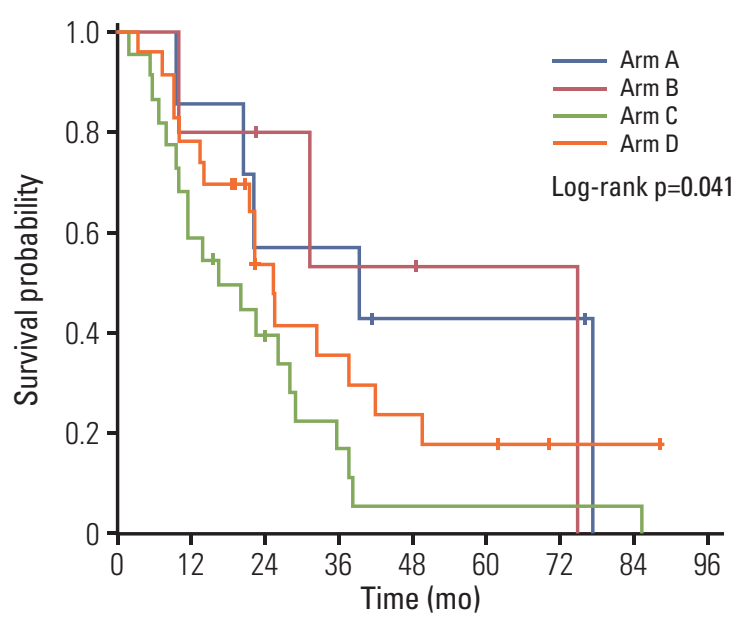

B

Fig. 2. Survival outcomes by treatment arm. (A) Progression-free survival (PFS). (B) Overall survival (OS).

\section{Patient characteristics}

Patient characteristics are shown in Table 1. Age, ECOG PS, stage, and involvement of supraclavicular lymph node were well balanced among the four arms. About $70 \%$ had stage IIIB disease and about $40 \%$ had supraclavicular lymph node involvement. As expected, the proportion of female, never smoker, and patients with adenocarcinoma was higher in the EGFR-mutant groups (arm A and B), but lower in the
EGFR wild-type or unknown groups (arm C and D). The patients with EGFR-unknown tumors were more likely to have squamous cell carcinoma histology than those with EGFR wild-type and mutant tumor (70.6\% vs. $30.0 \%$ vs. $8.3 \%$, $\mathrm{p}<0.001)$.

\section{Efficacy}

After induction therapy, the ORR was $71.4 \%$ in arm A, 
Table 3. Severe adverse effects

\begin{tabular}{|c|c|c|c|c|c|c|c|}
\hline \multirow[t]{2}{*}{ Adverse effect } & \multicolumn{3}{|c|}{$\begin{array}{l}\text { During induction therapy } \\
\text { z grade } 3(\%)\end{array}$} & \multicolumn{4}{|c|}{$\begin{array}{c}\text { During and after chemoradiotherapy } \\
\qquad \text { grade } 3(\%)\end{array}$} \\
\hline & Arm A & Arm B & Arm C & Arm A & Arm B & Arm C & Arm D \\
\hline Anemia & 0.0 & 0.0 & 13.6 & 0.0 & 0.0 & 22.2 & 4.2 \\
\hline Neutropenia & 0.0 & 0.0 & 50.0 & 0.0 & 20.0 & 72.2 & 41.7 \\
\hline Febrile neutropenia & 0.0 & 0.0 & 0.0 & 0.0 & 0.0 & 0.0 & 4.2 \\
\hline Thrombocytopenia & 0.0 & 0.0 & 4.5 & 0.0 & 0.0 & 11.1 & 4.2 \\
\hline AST/ ALT increased & 0.0 & 0.0 & 0.0 & 0.0 & 0.0 & 0.0 & 0.0 \\
\hline Creatinine increased & 0.0 & 0.0 & 4.5 & 0.0 & 0.0 & 0.0 & 0.0 \\
\hline Fatigue/Asthenia & 0.0 & 0.0 & 0.0 & 0.0 & 20.0 & 0.0 & 12.5 \\
\hline Anorexia & 0.0 & 0.0 & 0.0 & 0.0 & 20.0 & 0.0 & 4.2 \\
\hline Nausea & 0.0 & 0.0 & 0.0 & 0.0 & 0.0 & 0.0 & 4.2 \\
\hline Vomiting & 0.0 & 0.0 & 0.0 & 0.0 & 0.0 & 0.0 & 0.0 \\
\hline Skin rash & 14.3 & 20.0 & 0.0 & 0.0 & 0.0 & 0.0 & 0.0 \\
\hline Diarrhea & 0.0 & 0.0 & 4.5 & 0.0 & 0.0 & 5.6 & 0.0 \\
\hline Oral mucositis & 0.0 & 0.0 & 0.0 & 0.0 & 0.0 & 0.0 & 0.0 \\
\hline Radiation esophagitis & - & - & - & 0.0 & 20.0 & 0.0 & 4.2 \\
\hline Radiation pneumonitis & - & - & - & 0.0 & 0.0 & 5.6 & 8.7 \\
\hline
\end{tabular}

AST, aspartate aminotransferase; ALT, alanine transaminase.

$80.0 \%$ in arm B, and $70.0 \%$ in arm C (S2 Table). The best ORR after CCRT was $71.4 \%$ in arm A, $80.0 \%$ in arm B, $70.0 \%$ in arm C, and $73.9 \%$ in arm D (Table 2). According to the EGFR mutation status, the best ORR after CCRT was $75.0 \%$ in the EGFR-mutant type group, $80.8 \%$ in the EGFR-wild type group, and $58.8 \%$ in the EGFR-unknown group (S3 Table). Of 12 patients with EGFR-mutant tumors, nine showed PR as the best ORR, but one patient (exon 20 insertion) did show stable disease and two patients (exon 19 deletion and exon 20 insertion) had progressive disease (PD).

Median follow-up duration of the 13 surviving patients was 23.6 months (range, 15.3 to 88.2). There was no difference in PFS among treatment arms, with a median PFS of 11.6 months (95\% CI, 0.1 to 23.2) in arm A, 8.1 months (95\% CI, 2.7 to 13.6$)$ in arm B, 9.0 months (95\% CI, 7.5 to 10.4) in arm C, and 12.3 months (95\% CI, 6.6 to 18.0) in arm D ( $\mathrm{p}=0.134$ ) (Fig. 2). In terms of OS, the median OS was 39.3 months (95\% CI, 0.7 to 83.3 ) versus 31.2 months (95\% CI, 0.1 to 90.2 ) for arm A vs. arm B ( $\mathrm{p}=0.442)$ and 16.3 months $(95 \% \mathrm{CI}, 3.8$ to 28.9 ) versus 25.3 months (95\% CI, 20.8 to 29.8 ) for arm $C$ vs. $\operatorname{arm} \mathrm{D}(\mathrm{p}=0.050)$ (S4 Fig.). There was a trend towards longer OS in the EGFR-mutant groups than the EGFR wild-type or unknown groups: median OS, 39.3 months $(95 \% \mathrm{CI}, 0.1$ to $89.6)$ versus 22.1 months (95\% CI, 16.0 to 28.1$)$, respectively ( $p=0.081$ ) (S5 Fig.). When OS was compared between sensitive EGFR-mutant and wild-type groups, the median OS remained longer in patients with sensitive EGFR-mutant tumor than those with EGFR wild-type tumor (74.8 months [95\% CI, 5.4 to 144.2 ] vs. 25.3 months [95\% CI, 20.1 to 30.6]), respectively ( $\mathrm{p}=0.034)$.

Of 11 patients with EGFR-mutant tumors experiencing PD, 10 received subsequent treatment (S6 Table). Eight of these patients received EGFR-TKI, six as a second-line treatment and two as a third line treatment after intervening chemotherapy. The ORR after EGFR-TKI treatment was 75\% and the median PFS was 8.4 months (95\% CI, 2.0 to 14.8). The median survival after PD was significantly longer in the EGFR-mutant groups than the EGFR wild-type or unknown groups: 25.4 months (95\% CI, 19.5 to 31.5$)$ versus 9.8 months (95\% CI, 4.9 to 14.6 ), respectively ( $\mathrm{p}=0.017$ ).

\section{Toxicities}

There was no treatment-related mortality among all patients and all treatment regimens were tolerable. Table 3 lists the severe hematologic and non-hematologic toxicities during the induction phase and during or after the CCRT phase. Grade 3 or 4 neutropenia developed more frequently, but not febrile neutropenia in patients treated with IP relative to those treated with erlotinib. There was no difference in the incidence of grade 3 or 4 radiation pneumonitis between CCRT with erlotinib and CCRT with IP (arm A vs. arm B to $\mathrm{D}, 0.0 \%$ vs. $6.5 \%, \mathrm{p}=0.227$ ). 


\section{Pattern of treatment failure}

At the time of study analysis, 44 treatment failure sites (74.6\%) were known (S7 Table). Of the 44 treatment failures, the rates of loco-regional failure and distant failure were $36.3 \%$ and $47.7 \%$, respectively. Among distant failure sites, lung and brain were the two most common. There was no significant difference in loco-regional or distant failure rates among the four arms. In the EGFR-mutant groups, the locoregional and distant failure rates were $27.3 \%$ and $63.3 \%$, respectively, while they were $39.4 \%$ and $42.4 \%$ in the EGFR wild-type or unknown groups ( $\mathrm{p}=0.463$ ). The brain was more common as the first relapse site in the EGFR-mutant groups compared with the EGFR wild-type or unknown groups, even though there was no statistical significant difference between groups ( $45.5 \%$ vs. $18.2 \%$, $\mathrm{p}=0.070$ ).

\section{Discussion}

To the best of our knowledge, this was the first randomized phase II study to incorporate tailored chemotherapy regimens into the CCRT setting according to EGFR mutation status for unresectable stage III NSCLC patients. Although this study was closed early because of slow accrual, the results suggested that the combined-modality treatment based on molecular diagnostics was feasible for unresectable stage III NSCLC patients. In addition, concurrent administration of erlotinib or irinotecan plus cisplatin together with thoracic RT was safe and well tolerated without unexpected adverse events.

The results demonstrated that specific subsets such as female, never smoker, and patients with adenocarcinoma had a high likelihood of carrying an EGFR mutation in stage III lung cancer, which is consistent with stage IV lung cancer [17]. Patients with EGFR-mutant tumor showed similar ORR and median PFS, but longer median OS when compared to patients with EGFR-wild type tumor. The discordance between OS and PFS was caused by effective systemic therapy, namely EGFR-TKI, applied to patients with EGFRmutant tumor when the disease progressed after CCRT. Actually, the post-progression survival was significantly longer in patients with EGFR-mutant tumor compared to the other patients $(\mathrm{p}=0.017)$. Of the 12 patients having EGFRmutant tumor, 11 experienced PD and eight received EGFRTKIs during the disease course after PD. Their ORR to subsequent EGFR-TKIs was 75\% with a median PFS of 8.4 months, which was comparable to the clinical outcome of EGFR-TKIs in chemo-naive patients with stage IIIB/IV EGFR-mutant tumor [18-20]. In this study, erlotinib was only given during three cycles of induction therapy for arm $B$ and 11 cycles for arm A (three cycles of induction therapy, two cycles during and six cycles after CCRT). Thus, these groups appear to have remained sensitive to EGFR-TKIs at the time of progression and reuse of EGFR-TKIs was confirmed to be beneficial.

Approximately half of the patients with EGFR-mutant tumors presented with brain metastasis as the first site of treatment failure. The brain failure rate of this EGFR-mutant group was higher than the 15\%-30\% rate reported for unselected locally advanced NSCLC patients receiving standard curative multimodality treatment in previous studies [21-24]. This finding indicates that incorporation of EGFR-TKIs resulted in much better control of extracranial disease and increased survival for patients with EGFR-mutant tumor. Additionally, a high intrinsic potential for brain metastasis of EGFR-mutant tumor or incomplete drug penetration of the brain-blood barrier of EGFR-TKIs may explain the high incidence of brain failure in this patient group. Taken together, patients with stage III EGFR-mutant NSCLC may represent a distinct clinical subset, which warrants careful consideration in CCRT trial design and outcome evaluation.

When this study was designed, there were concerns about the possibility of EGFR-TKIs aggravating the radiationinduced pneumonitis because severe drug-induced interstitial lung disease is the most concerning toxicity of EGFRTKIs, with an incidence of about $1 \%$ [25]. In a Japanese clinical trial, 38 patients with stage III adenocarcinoma received $250 \mathrm{mg}$ gefitinib daily with thoracic $\mathrm{RT}$ after induction therapy and one of 35 patients stopped CCRT due to grade 3 pneumonitis [26]. In another prospective study, 49 stage III NSCLC patients whose ethnicity was mainly white received erlotinib $150 \mathrm{mg}$ daily for 6 days a week during thoracic $\mathrm{RT}$, but only three of 46 patients showed severe pneumonitis (two with grade 3 and one with grade 4) [27]. Although the subject numbers were small, our study also suggested that erlotinib before or during thoracic RT does not increase the incidence and severity of pneumonitis and can be safely added to CCRT.

We also tried to develop a more effective treatment schedule to control occult micrometastasis by applying a new chemotherapy regimen at full dose before or after CCRT in patients with EGFR unknown or wild-type tumor. This study showed there was no difference in the toxicity profile between the two treatment arms, but the consolidation treatment arm (arm D) showed longer survival than the induction treatment arm ( $\operatorname{arm} \mathrm{C})$, even though the sample size was too small to draw a firm conclusion. The survival outcome of the consolidation arm seems favorable, even when compared to those of historical randomized trials, which rarely exceeded 17 months after CCRT [4,5]. These results were consistent with those of other previous randomized phase II studies 
[28], suggesting that upfront CCRT followed by chemotherapy may be superior to chemotherapy followed by CCRT when both treatment strategies can achieve high completion rate. However, these results need to be confirmed in large randomized studies.

Among patients with unresectable stage III NSCLC, those carrying EGFR-mutant tumors might show a distinct clinical course with longer survival in contrast to other subsets. Thus, it is necessary to allocate appropriate treatment regimens to stage III NSCLC patients based on molecular characteristics in the era of molecular-targeted therapy. This randomized phase II study demonstrated that this treatment approach is feasible and well tolerated in this population.

\section{Electronic Supplementary Material}

Supplementary materials are available at Cancer Research and Treatment website (http://www.e-crt.org).

\section{Conflicts of Interest}

Erlotinib and irinotecan were provided by Roche Korea and Pfizer, Inc., respectively.

\section{Acknowledgments}

The study was supported in part by Roche Korea Co., Ltd. and a National Cancer Center Research grant (1410540).

\section{References}

1. Jemal A, Bray F, Center MM, Ferlay J, Ward E, Forman D. Global cancer statistics. CA Cancer J Clin. 2011;61:69-90.

2. Oh CM, Won YJ, Jung KW, Kong HJ, Cho H, Lee JK, et al. Cancerstatistics in Korea: incidence, mortality, survival, and prevalence in 2013. Cancer Res Treat. 2016;48:436-50.

3. Morgensztern D, Ng SH, Gao F, Govindan R. Trends in stage distribution for patients with non-small cell lung cancer: a National Cancer Database survey. J Thorac Oncol. 2010;5: 29-33.

4. Curran WJ Jr, Paulus R, Langer CJ, Komaki R, Lee JS, Hauser $S$, et al. Sequential vs. concurrent chemoradiation for stage III non-small cell lung cancer: randomized phase III trial RTOG 9410. J Natl Cancer Inst. 2011;103:1452-60.

5. Furuse K, Fukuoka M, Kawahara M, Nishikawa H, Takada Y, Kudoh S, et al. Phase III study of concurrent versus sequential thoracic radiotherapy in combination with mitomycin, vindesine, and cisplatin in unresectable stage III non-small-cell lung cancer. J Clin Oncol. 1999;17:2692-9.

6. Thatcher N, Chang A, Parikh P, Rodrigues Pereira J, Ciuleanu $\mathrm{T}$, von Pawel J, et al. Gefitinib plus best supportive care in previously treated patients with refractory advanced non-smallcell lung cancer: results from a randomised, placebo-controlled, multicentre study (Iressa Survival Evaluation in Lung Cancer). Lancet. 2005;366:1527-37.

7. Tamura K, Okamoto I, Kashii T, Negoro S, Hirashima T, Kudoh S, et al. Multicentre prospective phase II trial of gefitinib for advanced non-small cell lung cancer with epidermal growth factor receptor mutations: results of the West Japan Thoracic Oncology Group trial (WJTOG0403). Br J Cancer. 2008;98:907-14.

8. Lynch TJ, Bell DW, Sordella R, Gurubhagavatula S, Okimoto RA, Brannigan BW, et al. Activating mutations in the epidermal growth factor receptor underlying responsiveness of nonsmall-cell lung cancer to gefitinib. N Engl J Med. 2004;350:
2129-39.

9. Masuda N, Fukuoka M, Fujita A, Kurita Y, Tsuchiya S, Nagao $\mathrm{K}$, et al. A phase II trial of combination of CPT-11 and cisplatin for advanced non-small-cell lung cancer. CPT-11 Lung Cancer Study Group. Br J Cancer. 1998;78:251-6.

10. Mori K, Machida S, Yoshida T, Yoshida M, Kano Y, Tominaga $\mathrm{K}$. A phase II study of irinotecan and infusional cisplatin with recombinant human granulocyte colony-stimulating factor support for advanced non-small-cell lung cancer. Cancer Chemother Pharmacol. 1999;43:467-70.

11. Ueoka H, Tanimoto M, Kiura K, Tabata M, Takigawa N, Segawa $Y$, et al. Fractionated administration of irinotecan and cisplatin for treatment of non-small-cell lung cancer: a phase II study of Okayama Lung Cancer Study Group. Br J Cancer. 2001;85:9-13

12. Jagasia $\mathrm{MH}$, Langer CJ, Johnson DH, Yunus F, Rodgers JS, Schlabach LL, et al. Weekly irinotecan and cisplatin in advanced non-small cell lung cancer: a multicenter phase II study. Clin Cancer Res. 2001;7:68-73.

13. Han JY, Lim HS, Lee DH, Ju SY, Lee SY, Kim HY, et al. Randomized Phase II study of two opposite administration sequences of irinotecan and cisplatin in patients with advanced nonsmall cell lung carcinoma. Cancer. 2006;106:873-80.

14. Hanna N, Bunn PA Jr, Langer C, Einhorn L, Guthrie T Jr, Beck $\mathrm{T}$, et al. Randomized phase III trial comparing irinotecan/ cisplatin with etoposide/cisplatin in patients with previously untreated extensive-stage disease small-cell lung cancer. J Clin Oncol. 2006;24:2038-43.

15. Therasse P, Arbuck SG, Eisenhauer EA, Wanders J, Kaplan RS, Rubinstein L, et al. New guidelines to evaluate the response to treatment in solid tumors. European Organization for Research and Treatment of Cancer, National Cancer Institute of the United States, National Cancer Institute of Canada. J Natl Cancer Inst. 2000;92:205-16. 
16. Lee DH, Lee GK, Kong SY, Kook MC, Yang SK, Park SY, et al. Epidermal growth factor receptor status in anaplastic thyroid carcinoma. J Clin Pathol. 2007;60:881-4.

17. Shigematsu H, Gazdar AF. Somatic mutations of epidermal growth factor receptor signaling pathway in lung cancers. Int J Cancer. 2006;118:257-62.

18. Han JY, Park K, Kim SW, Lee DH, Kim HY, Kim HT, et al. First-SIGNAL: first-line single-agent iressa versus gemcitabine and cisplatin trial in never-smokers with adenocarcinoma of the lung. J Clin Oncol. 2012;30:1122-8.

19. Rosell R, Carcereny E, Gervais R, Vergnenegre A, Massuti B, Felip E, et al. Erlotinib versus standard chemotherapy as firstline treatment for European patients with advanced EGFR mutation-positive non-small-cell lung cancer (EURTAC): a multicentre, open-label, randomised phase 3 trial. Lancet Oncol. 2012;13:239-46.

20. Zhou C, Wu YL, Chen G, Feng J, Liu XQ, Wang C, et al. Erlotinib versus chemotherapy as first-line treatment for patients with advanced EGFR mutation-positive non-smallcell lung cancer (OPTIMAL, CTONG-0802): a multicentre, open-label, randomised, phase 3 study. Lancet Oncol. 2011;12: 735-42.

21. Cox JD, Scott CB, Byhardt RW, Emami B, Russell AH, Fu KK, et al. Addition of chemotherapy to radiation therapy alters failure patterns by cell type within non-small cell carcinoma of lung (NSCCL): analysis of radiation therapy oncology group (RTOG) trials. Int J Radiat Oncol Biol Phys. 1999;43: 505-9.

22. Andre F, Grunenwald D, Pujol JL, Girard P, Dujon A, Brouchet $\mathrm{L}$, et al. Patterns of relapse of N2 nonsmall-cell lung carcinoma patients treated with preoperative chemotherapy: should prophylactic cranial irradiation be reconsidered? Cancer. 2001;91:
2394-400

23. Law A, Karp DD, Dipetrillo T, Daly BT. Emergence of increased cerebral metastasis after high-dose preoperative radiotherapy with chemotherapy in patients with locally advanced nonsmall cell lung carcinoma. Cancer. 2001;92: $160-4$

24. Albain KS, Rusch VW, Crowley JJ, Rice TW, Turrisi AT 3rd, Weick JK, et al. Concurrent cisplatin/etoposide plus chest radiotherapy followed by surgery for stages IIIA (N2) and IIIB non-small-cell lung cancer: mature results of Southwest Oncology Group phase II study 8805. J Clin Oncol. 1995;13:1 880-92.

25. Qi WX, Sun YJ, Shen Z, Yao Y. Risk of interstitial lung disease associated with EGFR-TKIs in advanced non-small-cell lung cancer: a meta-analysis of 24 phase III clinical trials. J Chemother. 2015;27:40-51.

26. Niho S, Ohe Y, Ishikura S, Atagi S, Yokoyama A, Ichinose $Y$, et al. Induction chemotherapy followed by gefitinib and concurrent thoracic radiotherapy for unresectable locally advanced adenocarcinoma of the lung: a multicenter feasibility study (JCOG 0402). Ann Oncol. 2012;23:2253-8.

27. Komaki R, Allen PK, Wei X, Blumenschein GR, Tang X, Lee JJ, et al. Adding erlotinib to chemoradiation improves overall survival but not progression-free survival in stage III nonsmall cell lung cancer. Int J Radiat Oncol Biol Phys. 2015;92:317-24.

28. Belani CP, Choy H, Bonomi P, Scott C, Travis P, Haluschak J, et al. Combined chemoradiotherapy regimens of paclitaxel and carboplatin for locally advanced non-small-cell lung cancer: a randomized phase II locally advanced multi-modality protocol. J Clin Oncol. 2005;23:5883-91. 